\title{
Ferromagnetic Schottky Contact for GaN Based Spin Devices
}

\author{
TARKESHWAR C. PATIL \\ A 101, Jagruti Society, \\ Next to Abhishek Nursing Home, \\ Bhatwadi, Ghatkopar West, Mumbai 400084, Maharashtra \\ INDIA
}

\begin{abstract}
In this paper, ferromagnetic Schottky contacts for GaN based spin injection are being studied. The electrical characterization of this $\mathrm{Co} / \mathrm{n}-\mathrm{GaN}$ and $\mathrm{Fe} / \mathrm{n}-\mathrm{GaN}$ Schottky contacts showing the zero-bias barrier height comes closer to unity as the temperature is increased. Also, the Richardson constant is extracted for this Schottky contact. Both the zero-bias barrier height and the Richardson constant are verified both experimentally as well as theoretically. Thus, this Schottky contacts will serve as spin injector for GaN based spin devices specifically for $\mathrm{GaCrN}$ based devices.
\end{abstract}

Key-Words: - Schottky Contact; Ideality Factor; Gan; Spintronics; Ferromagnetism; Richardson Constant

Received: November 12, 2020. Revised: May 13, 2021. Accepted: June 1, 2021. Published: June 17, 2021.

\section{Introduction}

Spintronics is considered one of the potential technologies which can outperform the conventional semiconductor devices and bring in various functionalities allowing information processing and storage in fundamentally different and efficient ways. [1]

$\mathrm{GaN}$ is an extremely promising semiconductor material, for high-speed devices and many other applications like LEDs and lasers. It also has very small spin-orbit coupling due to its high bandgap, which may be useful for spintronic applications. The essential requirement for this is an optimized ferromagnet-GaN Schottky contact for efficient spin injection into GaN [2]. Recently, it is reported that $\mathrm{GaCrN}$ exhibits ferromagnetism at room temperature [3], therefore the spin injection in this material can be achieved using a ferromagnetic Schottky contact.

A) Cobalt (Co) is chosen as a spin polarization material and investigate for the first time the characteristics of Co/n-GaN Schottky contact. Some of the Schottky contact parameters like Schottky barrier height, ideality factor, and investigated the barrier inhomogeneity from temperature dependence of both Schottky barrier height and ideality factors which are extracted from current-voltage (I-V) data at different temperatures ranging from 300 to $470 \mathrm{~K}$ have been reported in this paper. It is observed that barrier height inhomogeneity which is found to match well with a Gaussian Distribution (GD). The extracted zero voltage mean barrier height and Richardson's constant with GD consideration are well matched with the predicted values. In the forward bias state, thermionic emission is found to be the dominant mode of carrier transport mechanism whereas electron tunneling may contribute significantly under reverse biased condition.

B) Ferrous $(\mathrm{Fe})$ is chosen as a high spin polarization material and investigate for the first time the characteristics of $\mathrm{Fe} / \mathrm{n}-\mathrm{GaN}$ Schottky diodes. Schottky barrier height, barrier inhomogeneity, and ideality factor from current-voltage $(I-V)$ and capacitance-voltage $(C-V)$ characteristics have calculated. The effect of temperature on these parameters have been studied. It is observed that barrier height inhomogeneity which is found to match well with a Gaussian Distribution (GD). We have also extracted Richardson's constant for these diodes. Though thermionic emission is found to be the dominant mode of carrier transport under forward bias condition, electron tunneling may contribute significantly under reverse biased condition.

\section{Experimental Details $2.1 \mathrm{Co} / \mathrm{n}-\mathrm{GaN}$}

The schematic diagram of Schottky contact used in our study is shown in Fig. 1(a). An undoped GaN buffer layer of $7 \mu \mathrm{m}$ is grown on sapphire $\left(\mathrm{Al}_{2} \mathrm{O}_{3}\right)$ substrate by hydride vapor-phase epitaxy (HVPE) 
followed by growth of Si-doped (n-type) GaN layer $(2 \mu \mathrm{m})$ with a doping density of $1-2 \times 10^{18} \mathrm{~cm}^{-3}$ by metal-organic-chemical-vapor-deposition

(MOCVD). The sample is then cleaned in organic solvent TCE, acetone and IPA for 5 minutes sonication each and dipped in high concentric HF solution for $90 \mathrm{sec}$ to remove native oxide. Then ohmic metal stack Ti $(20 \mathrm{~nm}) / \mathrm{Al}(150 \mathrm{~nm}) / \mathrm{Ni}(50$ $\mathrm{nm}) / \mathrm{Au}(125 \mathrm{~nm})$

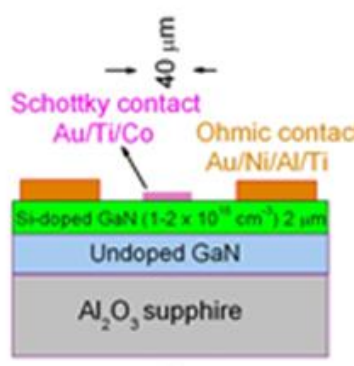

(a)

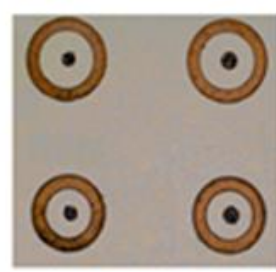

(b)
Fig. 1. a) Schematic diagram of fabricated device structure and (b) a micro - photograph of the devices are shown.

is deposited by electron-beam (e-beam) evaporation and annealing at $775^{\circ} \mathrm{C}$ using rapid thermal annealing (RTA) to realize the annular low resistance contact. Co $(30 \mathrm{~nm}) / \mathrm{Ti}(6 \mathrm{~nm}) / \mathrm{Au}(125$ $\mathrm{nm})$ Schottky contacts $40 \mu \mathrm{m}$ in diameter are then deposited at the center by e-beam evaporation. A microphotograph of the device is shown in Fig. 1(b).

\subsection{Fe/n-GaN}

The heterostructure of the sample used in our study is shown in Fig. 2(a). An undoped GaN buffer layer of $7 \mu \mathrm{m}$ is grown on sapphire $\left(\mathrm{Al}_{2} \mathrm{O}_{3}\right)$ substrate by hydride vapor-phase-epitaxy (HVPE). The Si-doped (n-type) GaN layer $(2 \mu \mathrm{m})$ is then grown by metalorganic-chemical-vapor-deposition (MOCVD) having a doping density of $1-2 \times 10^{18} \mathrm{~cm}^{-3}$. The sample is cleaned and native oxide is removed before depositing Ti $(20 \mathrm{~nm}) / \mathrm{Al}(150 \mathrm{~nm}) / \mathrm{Ni}(50$ $\mathrm{nm}) / \mathrm{Au}(125 \mathrm{~nm})$ metal stack by electron-beam (ebeam) evaporation and annealing at $775^{\circ} \mathrm{C}$ using rapid thermal annealing (RTA) for the annular low resistance ohmic contact. Fe $(50 \mathrm{~nm}) / \mathrm{Ti}(6 \mathrm{~nm}) /$ $\mathrm{Au}(125 \mathrm{~nm})$. Schottky contacts $60 \mu \mathrm{m}$ in diameter are then deposited at the center by e-beam evaporation. A micro-photograph of the device is shown in Fig. 2(b).

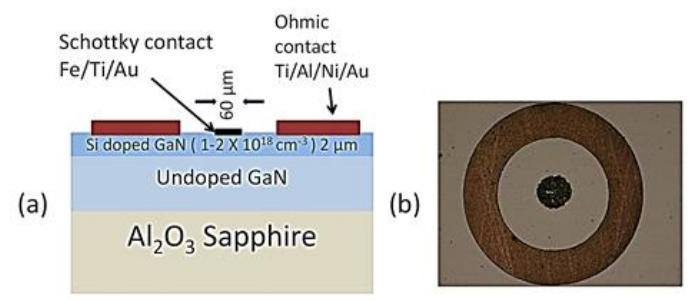

Fig. 2. a) Schematic diagram of fabricated device structure and (b) a micro - photograph of the devices are shown.

\section{Results and Discussions 3.1 Co/n-GaN}

Capacitance versus reverse bias voltage characteristics of the Schottky contact are measured at room temperature to extract the Schottky barrier height and doping concentration. For Schottky device, the reverse voltage dependent capacitance is given by [4],

$$
C^{-2}=2\left(\Phi_{B}+V-\frac{k T}{q}\right) /\left(q \epsilon_{s} S^{2} N_{D}\right)
$$

where $k$ is the Boltzmann constant, $q$ is the electronic charge, $s$ is dielectric constant of $\mathrm{GaN}$, and $S$ is effective area of Schottky contact. The barrier height $\left(\Phi_{B}\right)$ of $1.52 \mathrm{eV}$ and doping density $\left(N_{D}\right)$ of $1 \times 10^{18} \mathrm{~cm}^{-3}$ which is well matched with the nominal doping density are extracted from the extrapolation and slope of $C^{-2}$ versus $V$ plot (shown in Fig. 3).

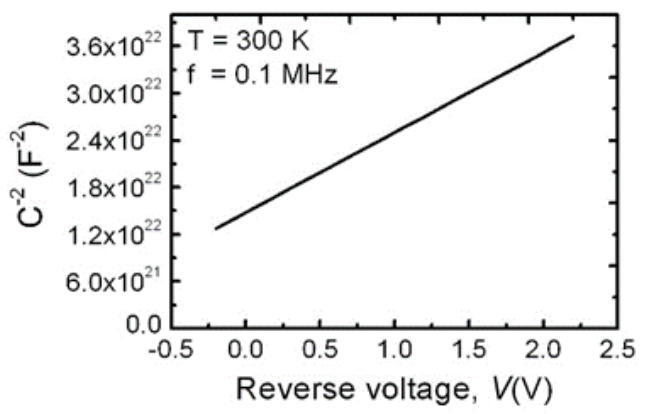

Fig. 3.C $\mathrm{C}^{-2}$ versus reverse voltage of Co/n-GaN Schottky contact at $300 \mathrm{~K}$

The temperature-dependent I-V characteristics of the contact can be seen in Fig. 4. It may be observed that the forward bias current is a strong a function of temperature, while the reverse bias current is relatively insensitive to changes in temperature. This indicates that thermionic emission is the dominant mechanism for electron transport from $\mathrm{GaN}$ to $\mathrm{Co}$ in forward bias and electron tunneling 
from Co to GaN may be the dominant process under reverse bias condition. The ideal thermionic emission model is used to extract both the zerovoltage-barrier-height and ideality factor using relation

$$
I=\left[S A^{*} T^{2} \exp \left(-\frac{q \Phi_{B 0}}{k T}\right)\right] \exp \left[\frac{q V}{n k T}-1\right]
$$

where $A^{*}$ is the Richardson's constant, $\Phi_{B 0}$ is the zero-voltage barrier height, and $n$ is the ideality factor.

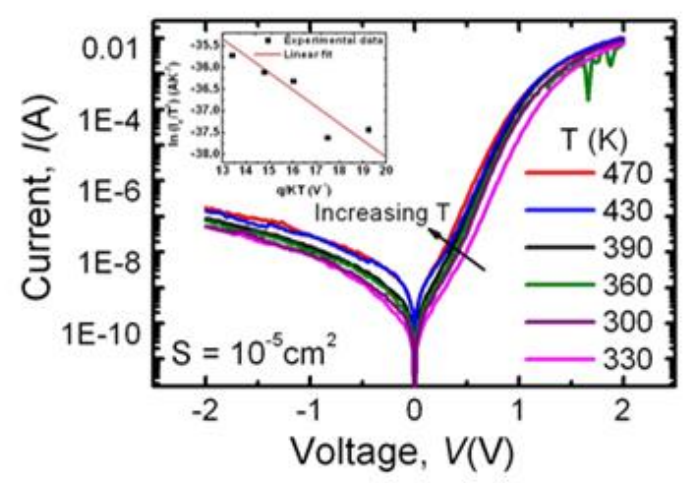

Fig. 4. Temperature dependent current versus voltage characteristics under forward and reverse bias conditions, inset figure shows the Richardson activation energy plot.

The voltage independent pre-factor in Eq. 2 , corresponding to the reverse saturation current $I_{0}$, is extracted from the linear region in the forwardbias $l n I$ vs. $V$ characteristics. $\Phi_{B 0}$ and $n$ as determined from experimental data and Eq. 2 show a strong variation with temperature varying from $0.82 \mathrm{eV}$ to $1.17 \mathrm{eV}$ and 2.26 to 1.4 , respectively, as the temperature increases from 300 to $475 \mathrm{~K}$ as mentioned in Fig. 5(a). The deviation from ideal Schottky behavior and the decrease of the barrier height at lower temperature might indicate barrier inhomogeneity or that other transport mechanisms are in play. With barrier inhomogeneity, current will be crowded in regions of lower barrier height, more so at lower temperature. Thus, increasing barrier height and more ideal Schottky behavior at higher temperature $[5,6]$ is explained.

The conventional Richardson's activation energy plot i.e. $\ln \left(I_{0} / T^{2}\right)$ versus $q / k T$ which should follow a linear relation is shown in inset of Fig. 4. The experimental data in the inset of Fig. 4 shows a deviation from linearity. This may be caused by barrier inhomogeneity and potential fluctuations at the $\mathrm{Co}-\mathrm{GaN}$ interface which translate to a temperature dependence of both phi and $\mathrm{n}$. The value of $A^{*}$ extracted from Fig. 4(a) is $2 \times 10^{-6} \mathrm{~A} \mathrm{~cm}^{-2} \mathrm{~K}^{-1}$ which is far away from the theoretically predicted value of $26.4 \mathrm{~A} \mathrm{~cm}^{-2} \mathrm{~K}^{-1}$.
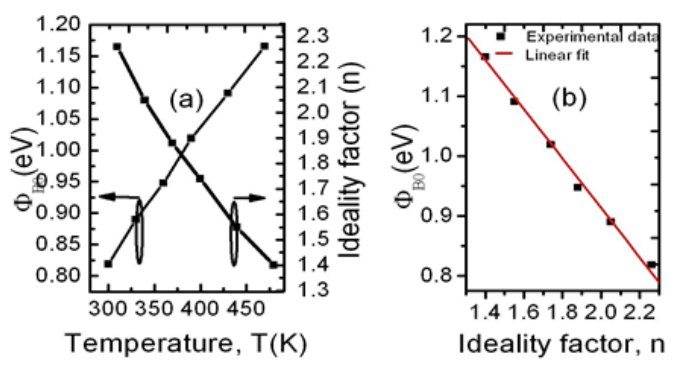

Fig. 5. (a) Zero bias barrier height and ideality factor as a function of temperature for $\mathrm{Co} / \mathrm{GaN}$ Schottky contact. (b) Linear dependency of zero bias barrier height on ideality factor.

Tung's theoretical approach used by

Schmitsdorf et al [7], has been followed to verify the presence of barrier inhomogeneity. According to them the barrier inhomogeneity results in a linear relation between $\bar{\Phi}_{B 0}$ and $n$. Fig. 5(b) shows the linear dependency of

extracted $\bar{\Phi}_{B 0}$ with n, confirming the presence of barrier inhomogeneity [7]. This implies that it is necessary to consider the barrier inhomogeneity in our analysis. To estimate the degree of barrier inhomogeneity, consider a

Gaussian distribution for barrier height having a mean value of $\bar{\Phi}_{B 0}$ and standard deviation $\sigma_{s}$ [8]. the Eq. 2 is modified as,

$I_{0}=\left[S A^{*} T^{2} \exp \left(-\frac{q}{k T}\left(\bar{\Phi}_{B 0}-\frac{q \sigma_{s}^{2}}{2 k T}\right)\right)\right]$

where $\bar{\Phi}_{B 0}$ is the zero bias mean barrier height. Fig. 6(a) shows that the zero-bias barrier height is linearly dependent on temperature, which confirms the presence of barrier inhomogeneity.

$\bar{\Phi}_{B 0}$ and $\sigma_{s}$ as determined from the plot are found to be $1.71 \mathrm{eV}$ and $0.23 \mathrm{eV}$, respectively. 

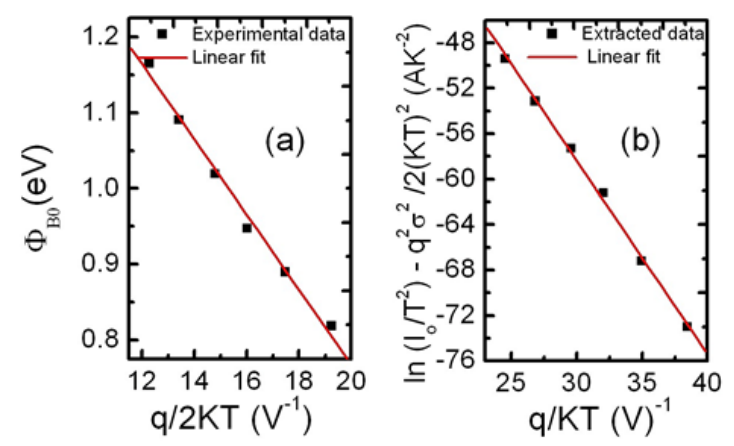

Fig. 6. (a) zero bias barrier height as a function of temperature for $\mathrm{Co} / \mathrm{n}-\mathrm{GaN}$ Schottky contact including the effect of barrier inhomogeneity. (b) Modified activation energy plot including barrier inhomogeneity.

It is seen that value of $\sigma_{s}$ compared to $\bar{\Phi}_{B 0}$, indicates significant barrier inhomogeneity in our contacts. The modified Richardson's plot to extract $A^{*}$ with the effect of barrier inhomogeneity is shown in Fig. 6(b). The value for $A^{*}$ is found to be $54 \mathrm{~A} \mathrm{~cm}^{-2} \mathrm{~K}^{-1}$, which is closer to the theoretical value. The value of

$\bar{\Phi}_{B 0}$ extracted here is $1.8 \mathrm{eV}$ which is reasonably close to the value obtained from $C-V$ analysis (Fig. 3).

\subsection{Fe/n-GaN}

Capacitance versus reverse bias voltage characteristics of the Schottky diodes are measured at $100 \mathrm{kHz}$ frequency for a temperature $(T)$ range of $300 \mathrm{~K}$ to $450 \mathrm{~K}$. The barrier height $\left(\Phi_{B}\right)$ and nominal doping density $\left(N_{D}\right)$ are extracted by plotting $1 / C^{2}$ versus $V$ (shown in Fig. 7) and using the expression [5],

$$
\frac{1}{C^{2}}=2\left(\Phi_{B}+V-\frac{k T}{q}\right) /\left(q \epsilon_{s} A^{2} N_{D}\right)
$$

where $k$ is the Boltzmann constant, $q$ is the electronic charge, $s$ is dielectric constant of $\mathrm{GaN}$, and $A$ is the area. It may be noted that both barrier height $\left(\Phi_{B}=1.3 \mathrm{eV}\right)$ and active dopant concentration $\left(N_{D}=1.5 \times 10^{18} \mathrm{~cm}^{-3}\right)$ change very little with temperature. The active dopant concentration matches well with the nominal doping density $1-2 \times 10^{18} \mathrm{~cm}^{-3}$.

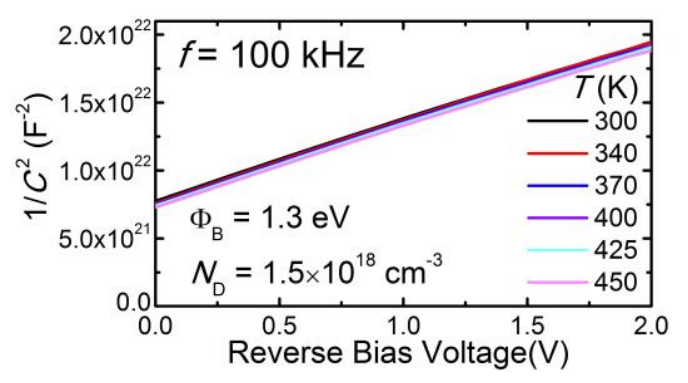

Fig. 7. Capacitance versus voltage characteristics of $\mathrm{Fe} / \mathrm{n}$ GaN Schottky diodes under reverse bias condition.

Figure. 8(a) shows the temperature dependent $I-V$ characteristics of the diode. While the forward bias characteristics is a strong a function of temperature, the reverse bias current is relatively insensitive to changes in temperature. This indicates that thermionic emission is the dominant mechanism for electron transport in forward bias and electron tunneling may be dominant process under reverse bias condition. Zero voltage barrier height and ideality factor (Fig. 8(b)) are determined as a function of temperature by considering thermionic emission under forward bias condition as

$$
\begin{aligned}
I=\left[A A^{*} T^{2} \exp (\right. & \left.\left.-\frac{q \Phi_{B 0}}{k T}\right)\right] \exp \left[\frac{q(V-I R)}{n k T}\right] \\
& \times\left(1-\exp \left[-\frac{q(V-I R)}{k T}\right]\right)
\end{aligned}
$$

where $A^{*}$ is the Richardson's constant, $\Phi_{B 0}$ is the zero-voltage barrier height, $R$ is the series resistance due to the semiconductor, and $n$ is the ideality factor. The voltage independent pre-
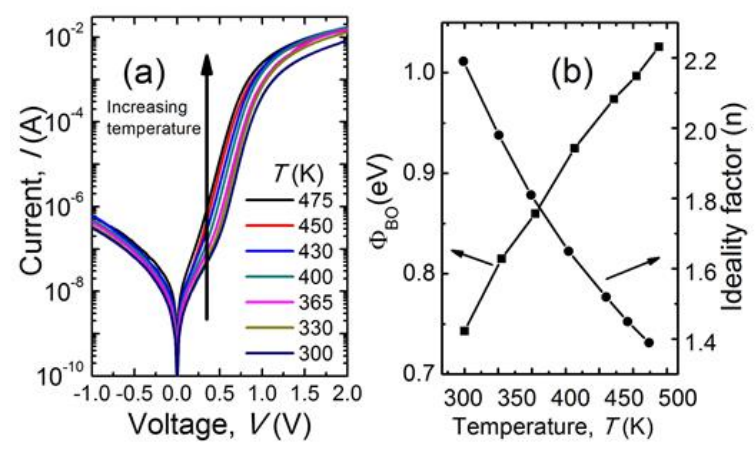

Fig. 8. (a) Temperature dependent current versus voltage characteristics under forward and reverse bias conditions; and (b) zero bias barrier height and ideality factor as a function of temperature for Fe/n-GaN Schottky diodes. 
factor in Eq. 5 corresponds to the reverse saturation current $I_{0} . \Phi_{B 0}$ and $n$ as determined from experimental data and Eq. 5 show a strong variation with temperature varying from 0.75 $\mathrm{eV}$ to $1.02 \mathrm{eV}$ and 2.2 to 1.4 , respectively, as the temperature increases from $300 \mathrm{~K}$ to $475 \mathrm{~K}$. The nonideal behavior and smaller barrier height at lower temperature regime indicate the possibility of other mechanism for electron transport and/or the presence of barrier inhomogeneity. In the presence of barrier inhomogeneity, the current will flow mostly through the regions of lower barrier height at low temperature where electrons having lower energy spread can surmount such barrier. This may explain the increasing barrier height and ideal Schottky diode behavior at higher temperatures.

Fig. 9(a) shows the conventional Richardson's activation energy plot i.e $\ln \left(I_{0} / T^{2}\right)$ versus $q / k T$ which should give a straight line. The data in Fig. 9(a) deviates from linearity because of the temperature dependence of both $\Phi_{B 0}$ and $n$ which arises possibly from barrier inhomogeneity and potential fluctuations at Fe$\mathrm{GaN}$ interface that consists of low and high barrier regions $[5,6]$.
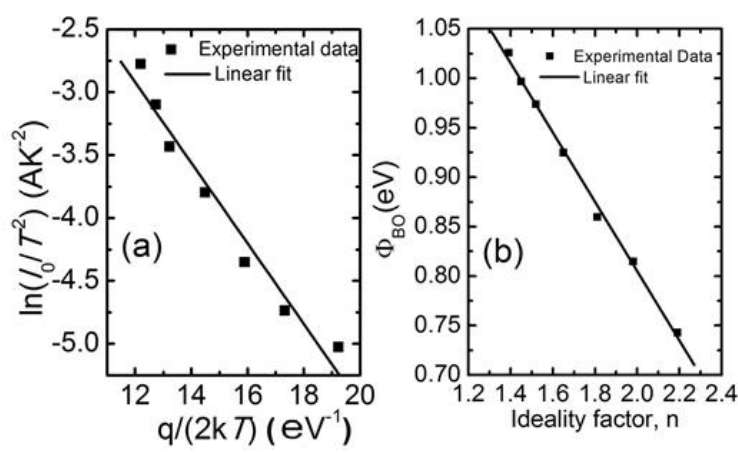

Fig. 9. (a) Determination of barrier height and Richardson's constant for Fe/n-GaN Schottky diodes; and (b) zero-bias barrier height as a function of ideality factor.

The value of $A^{*}$ extracted from Fig. 9(a) is $1.8 \times 10^{-7} \mathrm{~A} \mathrm{~cm}^{-2} \mathrm{~K}^{-1}$ which is far away from the theoretically predicted value of $26.4 \mathrm{~A} \mathrm{~cm}^{-2} \mathrm{~K}^{-1}$.

To ascertain the presence of barrier inhomogeneity, zero voltage barrier height is plotted as a function of ideality factor (Fig. 9(b)). The linear dependency confirms the presence of barrier inhomogeneity [7]. To estimate the degree of barrier inhomogeneity, we have considered a Gaussian distribution for

barrier height having a mean value of $\bar{\Phi}_{B 0}$ and standard deviation $\sigma$ s. It can be shown that Eq. 5 is then modified as,

$$
I_{0}=\left[A A^{*} T^{2} \exp \left(-\frac{q}{k T}\left(\bar{\Phi}_{B 0}-\frac{q \sigma_{s}^{2}}{2 k T}\right)\right)\right]
$$

where $\bar{\Phi}_{B 0}$ is the zero bias mean barrier height. Fig. 10(a) shows that the zero-bias barrier height is linearly dependent on temperature, which is indicative of the presence of barrier inhomogeneity. $\bar{\Phi}_{B 0}$ and $\sigma_{\mathrm{s}}$ as determined from the plot are found to be $1.52 \mathrm{eV}$ and $0.2 \mathrm{eV}$, respectively. The modified plot to determine
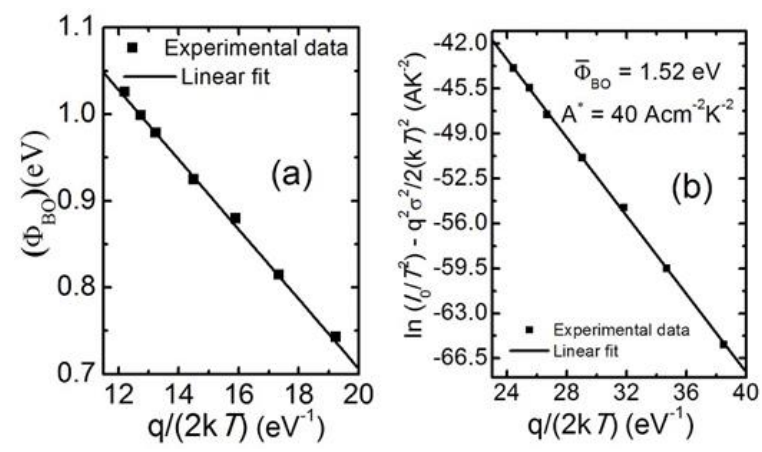

Fig. 10. (a) zero bias barrier height as a function of temperature for Fe/n-GaN Schottky diodes including the effect of barrier inhomogeneity. A linear dependency confirms the presence of such inhomogeneity and (b) Determination of zero bias mean barrier height and Richardson's constant from modified activation energy plot.

Richardson's constant including the effect of barrier inhomogeneity is shown in Fig. 10(b). The value for $A^{*}$ is found to be $40 \mathrm{~A} \mathrm{~cm}^{-2} \mathrm{~K}^{-1}$, which is closer to the predicted value. The value of $\bar{\Phi}_{B 0}$ extracted here is $1.52 \mathrm{eV}$ which is reasonably close to the value obtained from $\mathrm{C}$ $\mathrm{V}$ analysis (Fig. 7). 


\section{Conclusion}

\subsection{Co/n-GaN}

In summary, both experimentally and theoretically $\mathrm{Co} / \mathrm{n}-\mathrm{GaN}$ Schottky contacts have been investigated. The $I-V$ and $C-V$ characteristics of these devices were measured. It is observed that the zero-bias barrier height increases and ideality factor comes closer to unity with increasing temperature. It is also found that barrier inhomogeneity at the interface which may be due to the presence of interface gap states at $\mathrm{Co} / \mathrm{GaN}$ junction causes deviation from ideal characteristics. The extracted mean barrier height as well as Richardson's constant of GaN from modified activation energy plot considering a Gaussian distribution in the barrier heights is reported herein. The estimated mean barrier height and Richardson's constant is found to be close to the value obtained from $C-V$ analysis and theoretically predicted value respectively.

\subsection{Fe/n-GaN}

Both experimentally and theoretically $\mathrm{Fe} / \mathrm{n}-\mathrm{GaN}$ Schottky diodes were investigated. IV and C-V characteristics of these devices were carried out. It is observed that the zero-bias barrier height increases and ideality factor comes closer to unity with increasing temperature. It is found that barrier inhomogeneity at the interface causes deviation from ideal characteristics. The mean barrier height and Richardson's constant were extracted by considering the Gaussian distribution in the barrier heights. The estimated Richardson's constant is found to be close to the theoretically predicted value.

\section{References:}

[1] J. Fabian, A. Matos-Abiague, C. Ertler, P. Stano, and I. Žutić, "Semiconductor Spintronics" Acta Phys. Slov. vol. 57 No. 4 \& 5, 565 - 907 (2007) ISSN 1336-040X

[2] A. Fert and H. Jaffres, "Conditions for efficient spin injection from a ferromagnetic metal into a semiconductor" Phys. Rev. B vol. 64 , no. 18,184420 (2001) DOI: 10.1103/PhysRevB.64.184420
[3] T. Patil, " $\mathrm{Cl}_{2} / \mathrm{Ar}$ Based Dry Etching of GaCrN using Inductively Coupled Plasma," RSC Advances, vol. 6, no. 73, 68619-68626 (2016), DOI: 10.1039/C6RA13992B

[4] S. M. Sze, Physics of Semiconductor Devices, 2nd ed. (Willey, New York, 1981)

[5] S. Chand and J. Kumar, "Current-voltage characteristics and barrier parameters of $\mathrm{Pd} 2 \mathrm{Si} / \mathrm{p}-\mathrm{Si}$ (111) Schottky diodes in a wide temperature range" Semicond. Sci. Technol. vol. 10 , no. 12,1680 (1995) DOI: 10.1088/0268-1242/10/12/019

[6] M. S. P. Reddy, A. A. Kumar, V. R. Reddy, "Electrical transport characteristics of $\mathrm{Ni} / \mathrm{Pd} / \mathrm{n}-\mathrm{GaN}$ Schottky barrier diodes as a function of temperature" Thin Solid Films vol. 519, no. 11, 3844-3850 (2011) DOI: 10.1016/j.tsf.2011.01.258

[7] R. F. Schmitsdorf, T. U. Kampen and W. Monch "Correlation between Barrier Height and Interface Structure of $\mathrm{Ag} / \mathrm{Si}(111)$ Schottky Diodes," Surface Science, Vol. 324, No. 2-3, 249-256. (1995) DOI: 10.1016/0039-6028(94)00791-8

[8] S. Zhu, R.L.V. Meirhaeghe, C. Detavernier, F. Cardon, G. Ru, X. Qu, B. Li, "Barrier height inhomogeneities of epitaxial $\mathrm{CoSi} 2$ Schottky contacts on n-Si (100) and (111)," Solid-State Electronics, vol. 44, no. 4, 663671 (2000), DOI: 10.1016/S00381101(99)00268-3.

\section{Creative Commons Attribution License 4.0 (Attribution 4.0 International, CC BY 4.0)}

This article is published under the terms of the Creative Commons Attribution License 4.0 https://creativecommons.org/licenses/by/4.0/deed.en US 